\title{
Normal chest CT in 1091 symptomatic patients with confirmed Covid-19: frequency, characteristics and outcome
}

\author{
Ian Leonard-Lorant ${ }^{1}$ • François Severac ${ }^{2} \cdot$ Pascal Bilbault $^{3} \cdot$ Joris Muller $^{4} \cdot$ Pierre Leyendecker $^{1} \cdot$ Catherine Roy $^{1}$. \\ Mickaël Ohana ${ }^{1}$ if
}

Received: 5 August 2020 / Revised: 7 November 2020 / Accepted: 2 December 2020 / Published online: 13 January 2021

(C) European Society of Radiology 2021

\begin{abstract}
Objective Frequency of normal chest CT in symptomatic COVID-19 patients as well as the outcome of these patients remains unknown. The objectives of this work were to assess the incidence of initially normal chest CT in a cohort of consecutive confirmed COVID-19 patients with respiratory symptoms and to compare their clinical characteristics and their outcome to matched patients with typical COVID-19 lesions at initial CT.

Methods From March $6^{\text {th }}$ to April $22^{\text {nd }}$, all consecutive adult patients referred to the COVID-19 clinic of our Emergency Department were retrospectively analyzed. Each patient with a positive SARS-CoV-2 RT-PCR and a normal initial chest CT after second reading was 1:1 matched based on sex, age and date of CT acquisition to a patient with positive RT-PCR and initial chest CT with typical COVID-19 lesions. Clinical data, laboratory results and outcomes (major being mechanical ventilation and/ or death) were compared between both groups, using Wilcoxon signed-rank test, McNemar's chi-squared test and/or exact McNemar's test where appropriate.

Results Fifty-seven chest CT out of 1091 (5.2\%, 95\% CI 4.0-6.7) in symptomatic patients with positive RT-PCR were normal, with a median onset of symptoms of 4.5 days (IQR [1.25-10.25]). After a median follow-up of 43 days, death and/or mechanical ventilation occurred in 3 patients $(5.3 \%)$ in the study group, versus $11(19.3 \%)$ in the control group $(p=0.011)$.

Conclusions Normal initial chest CT occurred in 5.2\% of symptomatic confirmed COVID-19 cases in our cohort. While better than those with abnormal chest CT, outcome was not entirely benign with $5.3 \%$ death and/or mechanical ventilation.

Key Points

- In a cohort of 1091 symptomatic COVID-19 patients, initial chest CT was normal in 5.2\% of cases.

- Normal chest CT in confirmed COVID-19 is frequent even when onset of symptoms is greater than 3 days.

- The outcome of COVID-19 patients with initial normal chest CT, while better than those with abnormal CT, was not entirely benign with $5.3 \%$ death and/or mechanical ventilation.
\end{abstract}

Keywords Diagnostic imaging · Multidetector computed tomography · Lung diseases · COVID-19 · Reverse transcriptase polymerase chain reaction

Mickaël Ohana

mickael.ohana@gmail.com

1 Hôpitaux Universitaires de Strasbourg, Service de Radiologie, Nouvel Hôpital Civil, 1 place de l'Hôpital, 67000 Strasbourg, France

2 Hôpitaux Universitaires de Strasbourg, Groupe Méthodes en Recherche Clinique (GMRC), Hôpital Civil, Strasbourg, France

3 Hôpitaux Universitaires de Strasbourg, Service de Médecine d'Urgence, Nouvel Hôpital Civil, Strasbourg, France

4 Hôpitaux Universitaires de Strasbourg, Service de Santé Publique, Nouvel Hôpital Civil, Strasbourg, France

\author{
Abbreviations \\ CT Computed tomography \\ ED Emergency department \\ ICU Intensive care unit \\ RT-PCR Reverse transcriptase polymerase chain reaction
}

\section{Introduction}

COVID-19 infection in patients with respiratory symptoms usually manifests with characteristic chest CT lesions that have been broadly described in the literature [1-4], and whose 
extension correlates with disease severity and patients' outcome [5-7]. Yet, several publications have emphasized that chest $\mathrm{CT}$ could be and remain normal in a fraction of these symptomatic patients, particularly if acquired in the first 3 days after onset of symptoms, with varying incidence in small cohorts (20 out of 36 patients in [8], 1 out of 51 patients in [9] and 17 out of 149 patients in [10]).

Consequently, the exact frequency of normal chest $\mathrm{CT}$ in a homogeneous and large cohort of symptomatic COVID-19 patients as well as the outcome of these patients remains unknown. As chest CT can be used for triage of symptomatic patients suspected of COVID-19 [11-13], knowing the frequency and the impact of a normal chest $\mathrm{CT}$ at presentation in this population would be valuable to optimize their management and care.

The objectives of this work were therefore to assess the incidence of initially normal chest $\mathrm{CT}$ in a cohort of consecutive RT-PCR-confirmed COVID-19 patients with respiratory symptoms, and to compare their clinical characteristics as well as their outcome to matched COVID-19 patients with abnormal chest $\mathrm{CT}$.

\section{Patients and methods}

The local ethics committee of Strasbourg Hospital approved this retrospective study and waived the need for informed consent.

\section{COVID-19 pathway}

Starting March $6^{\text {th }}$, a dedicated pathway [14] for patients with a clinical suspicion of COVID-19 infection was set up within the Emergency Department (ED) of our single tertiary care centre (Nouvel Hôpital Civil, Strasbourg University Hospital, France). Symptomatic patients entering this pathway systematically underwent chest $\mathrm{CT}$ and nasopharyngeal swab at their arrival, to expedite subsequent triage made by the ED physician [11].

All patients who were symptomatic at presentation and went through this COVID-19 pathway from March $6^{\text {th }}$ to April $22^{\text {nd }}$ were included and were retrospectively analyzed when they had (a) a chest CT at the time of ED admission (i.e. initial chest CT present) and (b) at least one positive RT-PCR for SARS-CoV-2, with a delay between chest CT and first positive RT-PCR $\leq 48 \mathrm{~h}$ (i.e. confirmed COVID-19 infection).

\section{Chest CT}

Examinations were acquired on an 80-row scanner (Aquilion Prime SP, Canon Medical Systems), with parameters adjusted to the patient's morphotype (tension $100-135 \mathrm{kV}$ and maximum mAs 2-50). Images were reconstructed with a slice- thickness of $1 \mathrm{~mm}$ in mediastinal and parenchymal windows using an iterative reconstruction algorithm (AIDR-3D, Canon Medical Systems) and read on dedicated workstations with multiplanar and maximum intensity projection reconstructions.

Ten consultant radiologists (four specialized in chest imaging) with 5 to 30 years of experience were involved in the reading during the inclusion period. National Standards of reporting adapted from ESR and ESTI guidelines [1] were used and examinations were classified into one of 3 categories regarding the lung parenchyma findings:

- Category 1: highly suggestive of COVID-19 (bilateral and predominantly peripheral and subpleural ground glass opacities and/or alveolar consolidations);

- Category 2: indeterminate (bronchiolitis, centrilobular nodules, lobar consolidation);

- Category 3: normal examinations.

\section{RT-PCR}

Nasopharyngeal swab (Puritan Medical Products) was systematically performed at ED admission, concomitant to chest CT. Some patients had multiple sampling with either nasopharyngeal swab, sputum or bronchoalveolar lavage during their hospital stay. RT-PCR for SARS-CoV-2 was chosen as the reference standard and any positive result within $48 \mathrm{~h}$ of the initial chest $\mathrm{CT}$ was adjudicated as a confirmed COVID-19 infection.

\section{Study and control groups}

All patients with positive RT-PCR and normal chest CT (category 3 ) had their examinations reread independently by 2 thoracic radiologists to confirm the normality of the lung parenchyma; examinations with subtle parenchymal lesions in non-gravitational-dependent territories and consistent with minimal COVID-19 infection were excluded. The remaining patients constituted the study group, with strictly normal chest CT and RT-PCR-confirmed COVID-19.

Each patient of the study group was matched following a 1 to 1 fashion to a patient with a positive RT-PCR and a chest CT with typical COVID-19 lesions (category 1), to constitute the control group. Matching was based on sex, age ( \pm 1 year) and date of CT acquisition; if more than one control matched the characteristics of a patient from the study group, the control was randomly selected.

Sex, age, comorbidities, onset of initial symptoms and delay to CT, oxygenotherapy at ED admission, fever, lymphocyte rate, CRP, D-dimer, delay between CT scan to RT-PCR and follow-up CT (and delay between symptoms and FU-CT) were recorded for both groups. 
Clinical outcomes were obtained using electronic health records. Major events were of the need for mechanical ventilation and/or death; minor events were being hospitalized in a medicine ward and the length of hospital stay until discharge.

\section{Statistics}

Continuous variables are presented as medians with interquartile ranges (IQR) and were compared between both groups using the Wilcoxon signed-rank test. Categorical variables are presented as counts with percentages and were compared between both groups using McNemar's chi-squared test or exact McNemar's test where appropriate. The McNemar test was chosen since groups were not independent in this matched case-control study, and the statistical analysis of matched-pair studies must make allowance for the dependency in the data introduced by the matching, as underlined by Fagerland et al [15].

A $p$ value $<0.05$ was considered statistically significant.

All the analyses were performed using $\mathrm{R}$ software version 3.6.0. R Core Team (2019). R: A language and environment for statistical computing. R Foundation for Statistical Computing. URL https://www.R-project.org/.

\section{Results}

A flowchart of the study is shown in Fig. 1. All patients from the study group were matched to a control.

Ultimately, 57 chest CT out of 1091 (5.2\%, 95\% CI 4.06.7) in symptomatic patients with positive RT-PCR were normal, with a median onset of symptoms of 4.5 days (IQR [1.25-10.25]). Of note, 31 patients (54.4\%) had an onset of symptoms greater than 3 days and $13(17.5 \%)$ greater than 10 days. The median onset of symptoms was non-significantly higher in the control group, at 7 days (IQR [4-10]) ( $p=$ 0.115).

Patients in the study group required less oxygenotherapy support than in the control group $(p<0.001)$. Only $21 \%(n=$ 12 ) of patients with normal initial chest CT were febrile, compared to $65 \%$ of those with abnormal imaging $(n=37 ; p<$ $0.001)$. CRP values were also significantly lower (median $=$ $15 \mu \mathrm{g} / \mathrm{L}$ versus $51 \mu \mathrm{g} / \mathrm{L} ; p<0.001$ ), while D-dimer and lymphocyte median rates were similar in both groups.

A follow-up chest CT was performed only in 6 cases and remained normal in all but one patient.

Clinical data and outcomes of both groups are reported in Table 1.

After a median follow-up of 43 days, a major endpoint occurred in 3 patients in the study group (death secondary to respiratory insufficiency in 2 patients, mechanical ventilation in 1 patient), versus 11 patients in the control group (death secondary to respiratory insufficiency in 4 patients, mechanical ventilation in 7 patients) $(p=0.011)$.

\section{Discussion}

In a large cohort of consecutive symptomatic patients with positive RT-PCR, we found that initial chest CT was strictly normal in $5.2 \%$ of cases. Interestingly, normal chest CT was encountered in patients with variable onset of symptomsmore than 10 days in $17.5 \%$ of them. We also found that the outcome of these symptomatic patients, despite being significantly better than those with abnormal chest CT, is not entirely benign, with hospitalization needed in 16 patients $(28.1 \%)$ and death and/or mechanical ventilation occurring in $3(5.3 \%)$.

These results are complementary to the initial studies done on smaller cohorts [8-10], which however diverge on three significant points.

First, they reported an overall higher incidence of normal initial chest CT - up to 55\% in [8] in patients with early onset of symptoms. The use of a second independent reading by 2 chest radiologists in our work could partly explain this, since it enables to rule out 20 chest CT initially read as normal, by finding subtle subpleural ground glass opacities consistent with minimal COVID-19 infection.

Second, these studies suggested that most of the normal initial chest CT were encountered in the early phase of the disease, i.e. up to 3 days after the onset of symptoms. Our findings support that a negative chest CT can be encountered at all phases of the disease, and not only in the early one. Conversely, a negative chest CT in a patient symptomatic for more than 3 days cannot definitely rule out COVID-19.

Third, none of these studies reported the outcome of these patients. Since several studies have reported that patients with extensive lesions on chest CT had a worse prognosis [5, 6, 16], one might have thought that a normal chest could be a marker of a "benign" form of COVID-19. We found that the outcome of COVID-19 patients with an initially normal chest $\mathrm{CT}$, while better than those with abnormal CT, was not entirely benign with $28.1 \%$ of hospitalization with a median length of stay of 10 days, and $5.3 \%$ death and/or mechanical ventilation. Interestingly, while the rate of hospitalization was lower in these patients, the length of stay was not inferior to the one in the control group ( 8 days, $p=0.399$ ).

The frequency of strictly normal chest imaging in asymptomatic patients with confirmed COVID-19 also varies strongly in the literature, yet appears higher than what is reported in symptomatic patients. In 58 asymptomatic patients with RT-PCR-confirmed COVID-19, Meng et al found chest $\mathrm{CT}$ abnormalities in all patients, with no $\mathrm{CT}$ remaining normal [17]. These findings are counterbalanced by the report of 76 asymptomatic patients from the Diamond Princess, where Inui 


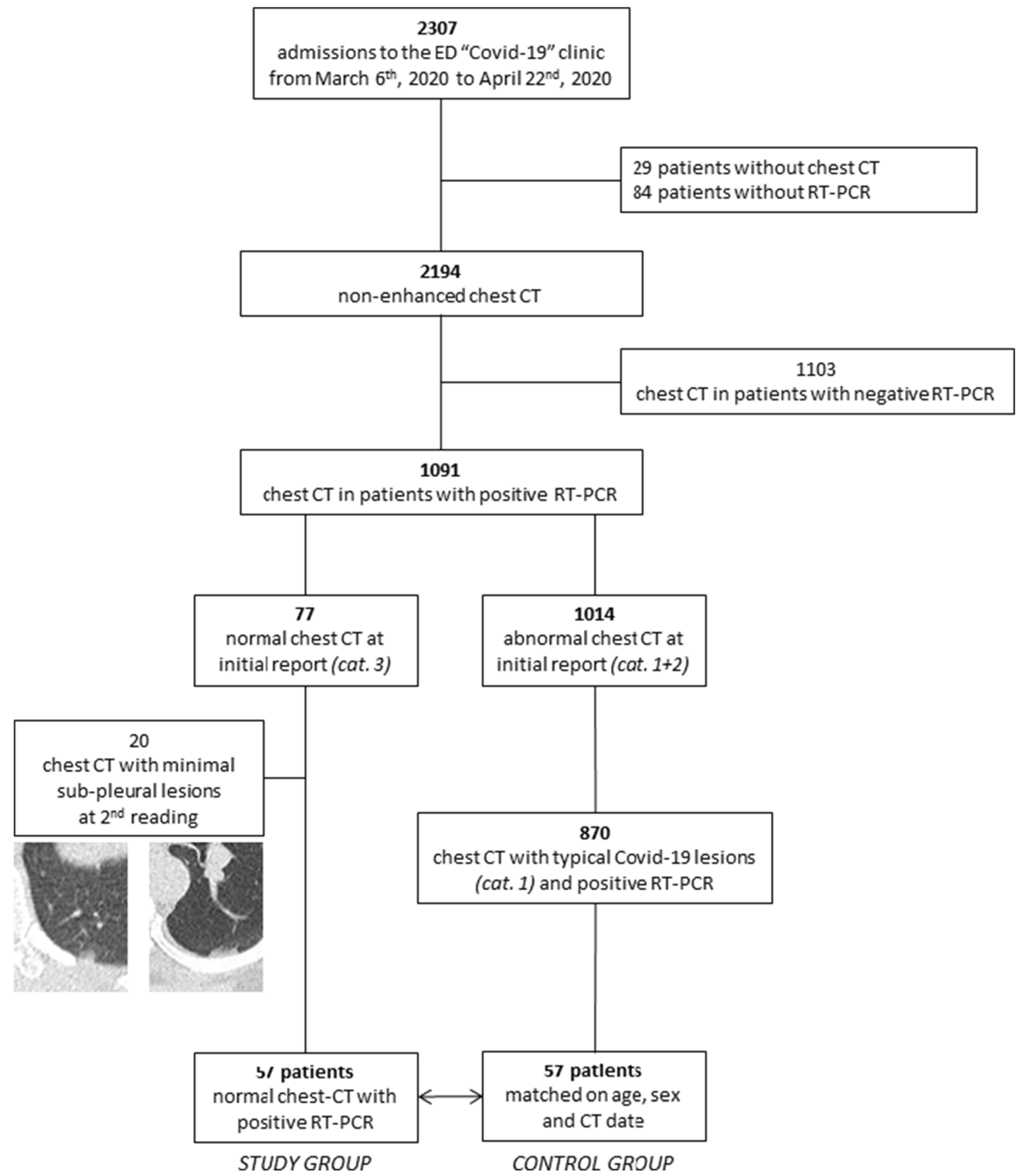

Fig. 1 Flowchart of the study. CT thumbnails are provided to illustrate subtle subpleural lesions that were missed at the first reading

et al found chest CT lesions in 41 of them, with 35 (46\%) chest $\mathrm{CT}$ remaining normal [18]. This range of normal examinations is also confirmed by Bandirali et al in 170 chest radiographs in asymptomatic patients, with 70 (41\%) remaining normal [19]. However, the outcome of these asymptomatic patients with normal imaging is not reported in these studies.

Our work has various limitations. First, matching was based only on age, sex and date of CT, and other confounding factors such as comorbidities or body mass index were not taken into account. Yet, older age and male sex were-by far-the two most prominent covariates associated with
COVID-19 death in a large scale UK study [20]. Second, only a fraction $(10.5 \%)$ of our population had a follow-up chest $\mathrm{CT}$; therefore, we cannot confirm that the initially normal chest $\mathrm{CT}$ remained normal in all patients during the course of the disease. Follow-up was ordered only in case of worsening symptoms, and yet it remained negative in $83 \%$ of cases. Third, our study is retrospective, even though we included all consecutive RT-PCR-positive cases with concomitant chest $\mathrm{CT}$, and therefore subject to some inclusion bias. Finally, this work was monocentric, from a hospital localized in an intense SARS-CoV-2 cluster and, as is, cannot be 
Table 1 Demographics, clinical data and outcome in the control and the study groups

\begin{tabular}{|c|c|c|c|c|c|}
\hline & \multicolumn{2}{|c|}{ CT-scan negative } & \multicolumn{3}{|c|}{ CT-scan positive } \\
\hline Patients $(n)$ & 57 & & 57 & & \\
\hline Male $(n)$ & 33 & $58 \%$ & 33 & $58 \%$ & \\
\hline Age—median IQR (years old) & \multicolumn{2}{|c|}{$41[29 ; 67]$} & \multicolumn{2}{|c|}{$41[29 ; 67]$} & $p=0.971$ \\
\hline Mean follow-up — median IQR (day) & \multicolumn{2}{|c|}{$43[26 ; 50]$} & \multicolumn{2}{|c|}{$43[37 ; 48]$} & $p=0.225$ \\
\hline Comorbidity & 32 & $56 \%$ & 27 & $47 \%$ & $p=0.340$ \\
\hline Cardiopathy, high blood pressure, atrial fibrillation & 9 & $16 \%$ & 3 & $5 \%$ & \\
\hline Neurodegenerative disease & 6 & $11 \%$ & 3 & $5 \%$ & \\
\hline Arteriopathy, stroke & 5 & $9 \%$ & 4 & $7 \%$ & \\
\hline Diabetes or dysthyroidism & 2 & $4 \%$ & 2 & $4 \%$ & \\
\hline Chronic obstructive pulmonary disease & 3 & $5 \%$ & 1 & $2 \%$ & \\
\hline Asthma & 1 & $2 \%$ & 2 & $4 \%$ & \\
\hline Morbid Obesity (BMI $\left.>40 \mathrm{~kg} / \mathrm{m}^{2}\right)$ & 1 & $2 \%$ & 4 & $7 \%$ & \\
\hline Dialysis or renal transplantation & \multicolumn{2}{|l|}{0} & 4 & $7 \%$ & \\
\hline Pregnancy & \multicolumn{2}{|l|}{0} & 1 & $2 \%$ & \\
\hline Other & 4 & $7 \%$ & 4 & $7 \%$ & \\
\hline Respiratory symptoms & 57 & $100 \%$ & 57 & $100 \%$ & \\
\hline Oxygenotherapy at CT scan & 6 & $11 \%$ & 31 & $54 \%$ & $p<0.001$ \\
\hline Digestive symptoms & 9 & $16 \%$ & 15 & $26 \%$ & \\
\hline Neurological symptoms & 16 & $28 \%$ & 10 & $18 \%$ & \\
\hline Fever & 12 & $21 \%$ & 37 & $65 \%$ & $p<0.001$ \\
\hline Lymphocyte rate — median IQR (G/L) & 1.46 & {$[1.00 ; 1.89]$} & 1.23 & {$[0.87 ; 1.74]$} & $p=0.112$ \\
\hline $\mathrm{CRP} —$ median IQR $(\mu \mathrm{g} / \mathrm{L}):$ normal range $<4 \mathrm{ug} / \mathrm{L}$ & \multicolumn{2}{|c|}{$n=56(98 \%)$} & \multicolumn{2}{|c|}{$n=55(96 \%)$} & $p<0.001$ \\
\hline D-dimer — median IQR $(\mathrm{g} / \mathrm{L})$ : normal range $<500 \mathrm{ug} / \mathrm{L}$ & \multicolumn{2}{|c|}{$n=22(39 \%)$} & \multicolumn{2}{|c|}{$n=20(35 \%)$} & $p=0.014$ \\
\hline Delay between symptom > CT scan (days) & 4.5 & {$[1.25 ; 10.25]$} & 7 & {$[4 ; 10]$} & $p=0.115$ \\
\hline Delay between CT scan > RT-PCR (days) & 0 & & 0 & & \\
\hline Follow-up CT scan $(n)$ & 6 & $11 \%$ & 7 & $12 \%$ & $p=0.340$ \\
\hline Delay between symptom > F-U CT scan (days) & 7 & & 21 & & \\
\hline Positive FU CT scan $(n)$ & 1 & $17 \%$ & 7 & $100 \%$ & \\
\hline Major endpoint & 3 & $5 \%$ & 11 & $19 \%$ & $p=0.011$ \\
\hline Death & 2 & $4 \%$ & 10 & $18 \%$ & \\
\hline Mechanical ventilation & 1 & $2 \%$ & 8 & $14 \%$ & \\
\hline Hospitalized in a COVID-19 ward & 16 & $28 \%$ & 40 & $70 \%$ & \\
\hline Hospitalization length—median IQR (in days) & 10 & {$[6.5 ; 15]$} & 8 & {$[6 ; 14]$} & $p=0.399$ \\
\hline Discharged home & 52 & $91 \%$ & 46 & $81 \%$ & $p=0.508$ \\
\hline
\end{tabular}

generalizable to other places with lesser prevalence of the disease, different populations or other medical systems. Particularly, hospital space was scarce during these times, and only severe or clinically concerning patients were admitted to medicine wards. Therefore, the rate of hospitalization in the present study might not reflect the usual standard of care.

To conclude, strictly normal chest CT occurred in $5.2 \%$ of symptomatic confirmed COVID-19 cases in our cohort, and while better than patients with abnormal chest CT, their outcome was not entirely benign. Close clinical follow-up of these patients is therefore to consider.
Acknowledgments The authors would like to thank Ms Sybille EICHERT for her precious help in the data collection.

Funding The authors state that this work has not received any funding.

\section{Compliance with ethical standards}

Guarantor The scientific guarantor of this publication is Pr Mickaël OHANA.

Conflict of interest The authors of this manuscript declare no relationships with any companies whose products or services may be related to the subject matter of the article. 
Statistics and biometry One of the authors has significant statistical expertise: Dr François SEVERAC is a biostatistician.

Informed consent Written informed consent was waived by the Institutional Review Board.

Ethical approval Institutional Review Board approval was obtained.

\section{Methodology}

- retrospective

- case-control

- performed at one institution

\section{References}

1. Revel MP, Parkar AP, Prosch H et al (2020) COVID-19 patients and the radiology department - advice from the European Society of Radiology (ESR) and the European Society of Thoracic Imaging (ESTI). Eur Radiol. https://doi.org/10.1007/s00330-020-06865-y

2. Hani C, Trieu NH, Saab I et al (2020) COVID-19 pneumonia: a review of typical CT findings and differential diagnosis. Diagn Interv Imaging 101(5):263-268

3. Jalaber C, Lapotre T, Morcet-Delattre T et al (2020) Chest CT in COVID-19 pneumonia: a review of current knowledge. Diagn Interv Imaging 101(7-8):431-437

4. Ojha V, Mani A, Pandey NN, Sharma S, Kumar S (2020) CT in coronavirus disease 2019 (COVID-19): a systematic review of chest CT findings in 4410 adult patients. Eur Radiol 30(11): 6129-6138

5. Yang R, Li X, Liu H et al (2020) Chest CT Severity score: an imaging tool for assessing severe COVID-19. Radiology: Cardiothoracic Imaging 2(2):e200047

6. Ruch Y, Kaeuffer C, Ohana M et al (2020) CT lung lesions as predictors of early death or ICU admission in COVID-19 patients. Clin Microbiol Infect. https://doi.org/10.1016/j.cmi.2020.07.030

7. Lanza E, Muglia R, Bolengo I et al (2020) Quantitative chest CT analysis in COVID-19 to predict the need for oxygenation support and intubation. Eur Radiol. https://doi.org/10.1007/s00330-02007013-2

8. Bernheim A, Mei X, Huang M et al (2020) Chest CT findings in Coronavirus Disease-19 (COVID-19): relationship to duration of infection. Radiology 295(3):200463. https://doi.org/10.1148/ radiol.2020200463
9. Fang Y, Zhang H, Xie J et al (2020) Sensitivity of Chest CT for COVID-19: comparison to RT-PCR. Radiology:200432. https:// doi.org/10.1148/radiol.2020200432

10. Yang W, Yan F (2020) Patients with RT-PCR-confirmed COVID19 and normal chest CT. Radiology 295(2):E3

11. Rubin GD, Ryerson CJ, Haramati LB et al (2020) The role of chest imaging in patient management during the COVID-19 pandemic: a multinational consensus statement from the Fleischner Society. Chest. https://doi.org/10.1016/j.chest.2020.04.003

12. Soyer P (2020) Lessons learned from chest CT in COVID-19. Diagn Interv Imaging 101(5):261-262

13. Ducray V, Vlachomitrou AS, Bouscambert-Duchamp $M$ et al (2020) Chest CT for rapid triage of patients in multiple emergency departments during COVID-19 epidemic: experience report from a large French university hospital. Eur Radiol. https://doi.org/10. 1007/s00330-020-07154-4

14. Oberlin M, Le Borgne P, Behr M, Kepka S, Bilbault P (2020) The organisation of a French emergency department in a coronavirus hotspot. Anaesth Crit Care Pain Med 39(4):457-458

15. Fagerland MW, Lydersen S, Laake P (2013) The McNemar test for binary matched-pairs data: mid-p and asymptotic are better than exact conditional. BMC Med Res Methodol 13:91

16. Li Y, Yang Z, Ai T, Wu S, Xia L (2020) Association of "initial CT" findings with mortality in older patients with coronavirus disease 2019 (COVID-19). Eur Radiol 30(11):6186-6193

17. Meng H, Xiong R, He R et al (2020) CT imaging and clinical course of asymptomatic cases with COVID-19 pneumonia at admission in Wuhan, China. J Infect. https://doi.org/10.1016/j.jinf. 2020.04.004

18. Inui S, Fujikawa A, Jitsu M et al (2020) Chest CT findings in cases from the cruise ship "Diamond Princess" with Coronavirus Disease 2019 (COVID-19). Radiology: Cardiothoracic Imaging 2(2): e200110

19. Bandirali M, Sconfienza LM, Serra R et al (2020) Chest radiograph findings in asymptomatic and minimally symptomatic quarantined patients in Codogno, Italy during COVID-19 pandemic. Radiology 295(3):E7

20. Williamson EJ, Walker AJ, Bhaskaran K et al (2020) OpenSAFELY: factors associated with COVID-19 death in 17 million patients. Nature. https://doi.org/10.1038/s41586-020$2521-4$

Publisher's note Springer Nature remains neutral with regard to jurisdictional claims in published maps and institutional affiliations. 